\title{
One more disproof for the Legendre's conjecture regarding the prime counting function $\pi(x)$
}

\author{
Reza Farhadian $^{1}$ and Rafael Jakimczuk ${ }^{2}$ \\ ${ }^{1}$ Department of Statistics, Lorestan University \\ Khorramabad, Iran \\ e-mail: farhadian.reza@yahoo.com \\ 2 División Matemática, Universidad Nacional de Luján \\ Buenos Aires, Argentina \\ e-mail: jakimczu@mail.unlu.edu.ar
}

Received: 12 October 2017

Accepted: 24 September 2018

Abstract: Let $\pi(x)$ denote the prime counting function, i.e., the number of primes not exceeding $x$. The Legendre's conjecture regarding the prime counting function states that

$$
\pi(x)=\frac{x}{\log x-A(x)},
$$

where Legendre conjectured that $\lim _{x \rightarrow \infty} A(x)=1.08366 \ldots$, which is the Legendre's constant. It is well-known that $\lim _{x \rightarrow \infty} A(x)=1$, and hence the Legendre's conjecture is not true. In this article we give various proofs of this limit and establish some generalizations.

Keywords: Primes, Prime counting function, Legendre's constant.

2010 Mathematics Subject Classification: Primary 11A41, Secondary 11A25, 11N05.

\section{Introduction}

The Prime Number Theorem (PNT) was first conjectured by the French mathematician AdrienMarie Legendre (1752-1833) as an experiential statement. In 1798, Legendre asserted that $\pi(x)=x /(A \log x-B)$ for constants $A$ and $B$ [6]. A decade latter, in 1808 [7] he refined his 
conjecture and formulated it by the form of

$$
\pi(x)=\frac{x}{\log x-A(x)},
$$

where Legendre conjectured that $A(x)$ is a function of $x$ such that

$$
\lim _{x \rightarrow \infty} A(x)=1.08366 \ldots
$$

which is the Legendre's constant.

In 1848, Chebyshev showed that if the function $A(x)$ tended to a limit as $x \rightarrow \infty$, then necessarily the limit had to be 1 [1]. In 2017, R. Farhadian obtained the numerical values of the function $A(x)$ at points $10^{k}$ for $k=1,2, \ldots, 25$, and then, based on the numerical observations, he noted that the function $A(x)$ decreases to 1 (not necessarily monotonically) [5]. In this article we give various proofs of the limit

$$
\lim _{x \rightarrow \infty} A(x)=1,
$$

proved by Vallée Poussin in 1899, and establish some generalizations.

\section{Main results}

By the Legendre's formula (1) we have

$$
A(x)=\log x-\frac{x}{\pi(x)} .
$$

We need the following lemmas.

\section{Lemma 2.1.}

$$
\frac{x}{\log x}\left(1+\frac{1}{\log x}+\frac{c_{1}}{\log ^{2} x}\right) \leq \pi(x) \leq \frac{x}{\log x}\left(1+\frac{1}{\log x}+\frac{c_{2}}{\log ^{2} x}\right),
$$

where $c_{1}=1.8$ for $x \geq 32299$ and $c_{2}=2.51$ for $x \geq 355991$.

Proof. See [3] and [4].

Lemma 2.2. If $k$ is a finite real number, then

$$
\lim _{x \rightarrow \infty} \frac{\log ^{3} x+k \log ^{2} x}{\log ^{3} x+\log ^{2} x+k \log x}=1 .
$$

Proof. We have

$$
\frac{\log ^{3} x+k \log ^{2} x}{\log ^{3} x+\log ^{2} x+k \log x}=\frac{1+\frac{k}{\log x}}{1+\frac{1}{\log x}+\frac{k}{\log ^{2} x}} .
$$


Theorem 2.3. Given the function $A(x)$ in relation (2). Then

$$
\lim _{x \rightarrow \infty} A(x)=1
$$

Besides, we have the inequality

$$
\frac{\log ^{3} x+1.8 \log ^{2} x}{\log ^{3} x+\log ^{2} x+1.8 \log x} \leq A(x) \leq \frac{\log ^{3} x+2.51 \log ^{2} x}{\log ^{3} x+\log ^{2} x+2.51 \log x},
$$

where the inequality on the left hand holds for $x \geq 32299$ and the inequality on the right hand holds for $x \geq 355991$.

Proof. By Lemma 1 we know that

$$
\frac{x}{\log x}\left(1+\frac{1}{\log x}+\frac{1.8}{\log ^{2} x}\right) \leq \pi(x) \leq \frac{x}{\log x}\left(1+\frac{1}{\log x}+\frac{2.51}{\log ^{2} x}\right) .
$$

By inverting the above inequality and multiplying the inequality by $x$, we have

$$
\frac{x}{\frac{x}{\log x}+\frac{x}{\log ^{2} x}+\frac{2.51 x}{\log ^{3} x}} \leq \frac{x}{\pi(x)} \leq \frac{x}{\frac{x}{\log x}+\frac{x}{\log ^{2} x}+\frac{1.8 x}{\log ^{3} x}},
$$

simplifying,

$$
\frac{\log x}{1+\frac{1}{\log x}+\frac{2.51}{\log ^{2} x}} \leq \frac{x}{\pi(x)} \leq \frac{\log x}{1+\frac{1}{\log x}+\frac{1.8}{\log ^{2} x}} .
$$

Clearly, if $\log x$ minus each part of the inequality (5), then we have (see equation (2))

$$
\log x-\frac{\log x}{1+\frac{1}{\log x}+\frac{1.8}{\log ^{2} x}} \leq A(x) \leq \log x-\frac{\log x}{1+\frac{1}{\log x}+\frac{2.51}{\log ^{2} x}} .
$$

Consequently

$$
\frac{\log ^{3} x+1.8 \log ^{2} x}{\log ^{3} x+\log ^{2} x+1.8 \log x} \leq A(x) \leq \frac{\log ^{3} x+2.51 \log ^{2} x}{\log ^{3} x+\log ^{2} x+2.51 \log x},
$$

that is, inequality (4). Finally, inequality (4) and Lemma 2.2 give limit (3).

In the following theorem we obtain an asymptotic formula for the function $A(x)$. An immediate corollary of this asymptotic formula is the $\operatorname{limit}_{x \rightarrow \infty} A(x)=1$.

Theorem 2.4. Let $h \geq 3$ be an arbitrary but fixed positive integer. The following asymptotic formula holds

$$
A(x)=\frac{1+\sum_{k=1}^{h-2} \frac{(k+1) !}{\log ^{k} x}+o\left(\frac{1}{\log ^{h-2} x}\right)}{1+\sum_{k=1}^{h-1} \frac{k !}{\log ^{k} x}+o\left(\frac{1}{\log ^{h-1} x}\right)} .
$$


Proof. The following asymptotic formula for the prime counting function $\pi(x)$ is well-known [8]

$$
\pi(x)=\sum_{k=1}^{h} \frac{(k-1) ! x}{\log ^{k} x}+o\left(\frac{x}{\log ^{h} x}\right),
$$

where $h$ is an arbitrary but fixed positive integer.

Therefore we have

$$
\frac{x}{\pi(x)}=\frac{\log x}{\sum_{k=1}^{h} \frac{(k-1) !}{\log ^{k-1} x}+o\left(\frac{1}{\log ^{h-1} x}\right)},
$$

and consequently

$$
\begin{aligned}
A(x)= & \log x-\frac{x}{\pi(x)}=\log x\left(1-\frac{1}{\sum_{k=1}^{h} \frac{(k-1) !}{\log ^{k-1} x}+o\left(\frac{1}{\log ^{h-1} x}\right)}\right) \\
= & \log x \frac{\sum_{k=2}^{h} \frac{(k-1) !}{\log ^{k-1} x}+o\left(\frac{1}{\log ^{h-1} x}\right)}{1+\sum_{k=2}^{h} \frac{(k-1) !}{\log ^{k-1} x}+o\left(\frac{1}{\log ^{h-1} x}\right)} \\
= & \frac{1+\sum_{k=3}^{h} \frac{(k-1) !}{\log ^{k-2} x}+o\left(\frac{1}{\log ^{h-2} x}\right)}{1+\sum_{k=2}^{h} \frac{(k-1) !}{\log ^{k-1} x}+o\left(\frac{1}{\log ^{h-1} x}\right)}=\frac{1+\sum_{k=1}^{h-2} \frac{(k+1) !}{\log ^{k} x}+o\left(\frac{1}{\log ^{h-2} x}\right)}{1+\sum_{k=1}^{h-1} \frac{k !}{\log ^{k} x}+o\left(\frac{1}{\log ^{h-1} x}\right)} .
\end{aligned}
$$

Now, we give other proof that $\lim _{x \rightarrow \infty} A(x)=1$ using formula for the $n$-th prime $p_{n}$ and $\log p_{n}$. We put $d_{n}=p_{n+1}-p_{n}$.

Theorem 2.5. The following limit holds

$$
\lim _{x \rightarrow \infty} A(x)=1 .
$$

Proof. The prime number theorem $p_{n} \sim n \log n$ implies the following formula for $\log p_{n}$

$$
\log p_{n}=\log n+\log \log n+o(1)
$$

On the other hand, a consequence of the equation (see (6) with $h=2$ )

$$
\pi(x)=\frac{x}{\log x}+\frac{x}{\log ^{2} x}+o\left(\frac{x}{\log ^{2} x}\right)
$$

is the following well-known formula for $p_{n}$ [2]

$$
p_{n}=n \log n+n \log \log n-n+o(n) .
$$

Therefore, we have (by (7) and (8))

$$
A\left(p_{n}\right)=\log p_{n}-\frac{p_{n}}{\pi\left(p_{n}\right)}=\log p_{n}-\frac{p_{n}}{n}=1+o(1)
$$


and consequently

$$
A\left(p_{n+1}\right)=\log p_{n+1}-\frac{p_{n+1}}{\pi\left(p_{n+1}\right)}=\log p_{n+1}-\frac{p_{n+1}}{n+1}=1+o(1) .
$$

Now, we have (by (9) and (10))

$$
\begin{gathered}
A\left(p_{n}\right)=A\left(p_{n+1}-d_{n}\right)=\log \left(p_{n+1}-d_{n}\right)-\frac{p_{n+1}-d_{n}}{n}=\log p_{n+1} \\
+\log \left(1-\frac{d_{n}}{p_{n+1}}\right)-\frac{p_{n+1}}{n+1}-\frac{p_{n+1}}{n(n+1)}+\frac{d_{n}}{n}=1+o(1)+\frac{d_{n}}{n}=1+o(1) .
\end{gathered}
$$

Therefore

$$
\frac{d_{n}}{n}=o(1)
$$

Let us consider an arbitrary sequence $a_{n}$ such that $\lim _{n \rightarrow \infty} a_{n}=\infty$. For each $n$ there is a prime $p_{n^{\prime}}$ such that $p_{n^{\prime}}<a_{n} \leq p_{n^{\prime}+1}$. Hence $a_{n}=p_{n^{\prime}+1}-b_{n^{\prime}}$, where $0 \leq b_{n^{\prime}}<d_{n^{\prime}}$. If in the sequence $a_{n}$ there is a subsequence of prime numbers $p_{n^{\prime}+1}$, we have (see above) $A\left(p_{n^{\prime}+1}\right)=1+o(1)$, therefore, we consider the subsequence of $a_{n}$ such that $a_{n} \neq p_{n^{\prime}+1}$ and consequently $\pi\left(a_{n}\right)=n^{\prime}$. For this subsequence we have

$$
A\left(a_{n}\right)=A\left(p_{n^{\prime}+1}-b_{n^{\prime}}\right)=\log \left(p_{n^{\prime}+1}-b_{n^{\prime}}\right)-\frac{p_{n^{\prime}+1}-b_{n^{\prime}}}{n^{\prime}}=\cdots=1+o(1) .
$$

Therefore for the complete sequence $a_{n}$ we have $A\left(a_{n}\right)=1+o(1)$. Consequently, by a wellknown theorem of analysis [9], we have $\lim _{x \rightarrow \infty} A(x)=1$.

Let $k$ be an arbitrary but fixed positive integer and let us consider the sequence $p_{n}^{k}$, that is, the sequence of the $k$-th powers of the prime numbers. In particular, if $k=1$, we obtain the sequence of primes; if $k=2$, we obtain the sequence of squares of primes, etc.

The Prime Number Theorem establishes

$$
\pi(x) \sim \frac{x}{\log x} .
$$

Let $\pi_{k}(x)$ be the number of $k$-th powers of primes not exceeding $x$, that is, $p_{n}^{k} \leq x$, then $\pi_{1}(x)=\pi(x)$. The Prime Number Theorem gives

$$
\pi_{k}(x)=\pi(\sqrt[k]{x}) \sim \frac{\sqrt[k]{x}}{\log \sqrt[k]{x}} .
$$

We have the following generalization of the Legendre's formula.

Theorem 2.6. Let $s$ and $k$ be arbitrary but fixed positive integers. We have

$$
\left(\pi_{k}(x)\right)^{s}=\frac{(\sqrt[k]{x})^{s}}{\log ^{s} \sqrt[k]{x}-A_{k, s}(x) \log ^{s-1} \sqrt[k]{x}},
$$

where $\lim _{x \rightarrow \infty} A_{k, s}(x)=s$. 
Proof. We have (see (6) with $h=2$ )

$$
\pi(x)=\frac{x}{\log x}+\frac{x}{\log ^{2} x}+o\left(\frac{x}{\log ^{2} x}\right) .
$$

Therefore

$$
\pi_{k}(x)=\pi(\sqrt[k]{x})=\frac{\sqrt[k]{x}}{\log \sqrt[k]{x}}+\frac{\sqrt[k]{x}}{\log ^{2} \sqrt[k]{x}}+o\left(\frac{\sqrt[k]{x}}{\log ^{2} \sqrt[k]{x}}\right)
$$

and consequently

$$
\begin{aligned}
\left(\pi_{k}(x)\right)^{s} & =\frac{(\sqrt[k]{x})^{s}}{\log ^{s} \sqrt[k]{x}}\left(1+\frac{1}{\log \sqrt[k]{x}}+o\left(\frac{1}{\log \sqrt[k]{x}}\right)\right)^{s} \\
& =\frac{(\sqrt[k]{x})^{s}}{\log ^{s} \sqrt[k]{x}}\left(1+\frac{s}{\log \sqrt[k]{x}}+o\left(\frac{1}{\log \sqrt[k]{x}}\right)\right)
\end{aligned}
$$

Now, we have

$$
\begin{aligned}
& A_{k, s}(x)=\log \sqrt[k]{x}-\frac{1}{\log ^{s-1} \sqrt[k]{x}} \frac{(\sqrt[k]{x})^{s}}{\left(\pi_{k}(x)\right)^{s}} \\
= & \log \sqrt[k]{x}-\frac{\log \sqrt[k]{x}}{1+\frac{s}{\log \sqrt[k]{x}}+o\left(\frac{1}{\log \sqrt[k]{x}}\right)}=\log \sqrt[k]{x} \frac{\frac{s}{\log \sqrt[k]{x}}+o\left(\frac{1}{\log \sqrt[k]{x}}\right)}{1+\frac{s}{\log \sqrt[k]{x}}+o\left(\frac{1}{\log \sqrt[k]{x}}\right)} \\
= & \frac{s+o(1)}{1+o(1)}=s+o(1) .
\end{aligned}
$$

Corollary 2.7. We have

$$
\pi_{k}(x)=\frac{\sqrt[k]{x}}{\log \sqrt[k]{x}-A_{k, 1}(x)},
$$

where $\lim _{x \rightarrow \infty} A_{k, 1}(x)=1$. Besides

$$
\lim _{x \rightarrow \infty} A(x)=1 .
$$

Proof. It is the case $s=1$ in the former theorem. Note that the limit 1 does not depend of $k$. On the other hand, $A(x)=A_{1,1}(x)$.

In the following theorem we establish other generalization of the Legendre's formula. We have the asymptotic formula (see (6))

$$
\pi(x)=\sum_{k=1}^{h} \frac{(k-1) ! x}{\log ^{k} x}+o\left(\frac{x}{\log ^{h} x}\right) .
$$

If $h=1$, we obtain the Prime Number Theorem

$$
\pi(x)=\frac{x}{\log x}+o\left(\frac{x}{\log x}\right) .
$$


The Legendre's formula is

$$
\pi(x)=\frac{x}{\log x-A(x)},
$$

where $\lim _{x \rightarrow \infty} A(x)=1$.

If $h=2$, we obtain

$$
\pi(x)=\frac{x}{\log x}+\frac{x}{\log ^{2} x}+o\left(\frac{x}{\log ^{2} x}\right) .
$$

If we put the formula

$$
\pi(x)=\frac{x}{\log x}+\frac{x}{\log ^{2} x-A_{2}(x) \log x},
$$

then we shall prove in the following theorem that $\lim _{x \rightarrow \infty} A_{2}(x)=2$, etc.

In general, we have the following theorem.

Theorem 2.8. Let $h \geq 2$ be an arbitrary but fixed positive integer. We have the following formula

$$
\pi(x)=\sum_{k=1}^{h-1} \frac{(k-1) ! x}{\log ^{k} x}+\frac{(h-1) ! x}{\log ^{h} x-A_{h}(x) \log ^{h-1} x},
$$

where

$$
\lim _{x \rightarrow \infty} A_{h}(x)=h .
$$

Proof: We have

$$
A_{h}(x)=\log x-\frac{1}{\log ^{h-1} x} \frac{(h-1) ! x}{\pi(x)-\sum_{k=1}^{h-1} \frac{(k-1) ! x}{\log ^{k} x}} .
$$

On the other hand, we have the equation

$$
\pi(x)=\sum_{k=1}^{h+2} \frac{(k-1) ! x}{\log ^{k} x}+o\left(\frac{x}{\log ^{h+2} x}\right) .
$$

Substituting this equation in the former equation we find that

$$
\begin{aligned}
& A_{h}(x)=\log x-\frac{1}{\log ^{h-1} x} \frac{(h-1) ! x}{\frac{(h-1) ! x}{\log ^{h} x}+\frac{h ! x}{\log ^{h+1} x}+\frac{(h+1) ! x}{\log ^{h+2} x}+o\left(\frac{x}{\log ^{h+2} x}\right)} \\
= & \log x\left(1-\frac{(h-1) !}{(h-1) !+\frac{h !}{\log x}+\frac{(h+1) !}{\log ^{2} x}+o\left(\frac{1}{\log ^{2} x}\right)}\right) \\
= & \frac{h !+\frac{(h+1) !}{\log x}+o\left(\frac{1}{\log x}\right)}{(h-1) !+\frac{h !}{\log x}+\frac{(h+1) !}{\log ^{2} x}+o\left(\frac{1}{\log ^{2} x}\right)}=h+o(1) .
\end{aligned}
$$


Cipolla's asymptotic formula for the $n$-th prime $p_{n}$ is [2]

$$
p_{n}=n \log n+n \log \log n-n+\sum_{i=1}^{r} \frac{(-1)^{i-1} n P_{i}(\log \log n)}{i ! \log ^{i} n}+o\left(\frac{n}{\log ^{r} n}\right),
$$

where $r$ is an arbitrary but fixed positive integer and $P_{i}(x)$ is a polynomial of degree $i$ and leading coefficient $(i-1)$ !.

Theorem 2.9. The following asymptotic formula holds

$$
\begin{aligned}
& p_{n}=n \log n+n \log \log n-n+\sum_{i=1}^{r-1} \frac{(-1)^{i-1} n P_{i}(\log \log n)}{i ! \log ^{i} n} \\
& +\quad(-1)^{r-1} \frac{n P_{r}(\log \log n)}{r ! \log ^{r} n+B_{r}(n) r ! \log ^{r-1} n \log \log n},
\end{aligned}
$$

where $\lim _{n \rightarrow \infty} B_{r}(n)=\frac{r}{r+1}$.

Proof. The proof is similar to the proof of the former theorem.

\section{Acknowledgements}

Rafael Jakimczuk is very grateful to Universidad Nacional de Luján.

\section{References}

[1] Chebyshev, P. L. (1851) Sur la fonction qui détermine la totalité des nombres premiers inférieurs à une limite donnée, Mémoires présentés à l'Académie Impériale des Sciences de St-Pétersbourg par divers Savants et lus dans ses Assemblées, Bd. 6, S., 141-157.

[2] Cipolla, M. (1902) La determinazione assintotica dell' $n^{i m o}$ numero primo, Rend. Acad. Sci. Fis. Mat. Napoli, Ser. 3, 8, 132-166.

[3] Dusart, P. (1998) Sharper bounds for $\phi, \theta, \pi$ and $p_{n}$, Rapport de recherche, Université de Limoges.

[4] Dusart, P. (1999) Inégalités explicites pour $\psi(X), \theta(X), \pi(X)$ et les nombres premiers, C. R. Math. Acad. Sci. Soc. R. Can., 21, 53-59.

[5] Farhadian, R. (2017) On a Conjecture of Legendre, MAYFEB Journal of Mathematics, 3, $53-58$.

[6] Legendre, A. M. (1798) Essai sur la Théorie de Nombres, 1st ed., Paris: Duprat, p. 19.

[7] Legendre, A. M. (1808) Essai sur la Théorie de Nombres, 2nd ed., Paris: Courcier, p. 394.

[8] LeVeque, J. W. (1958) Topics in Number Theory, Volume II, Addison-Wesley.

[9] Rey Pastor, J., Pi Calleja, P. \& Trejo, C. (1969) Análisis Matemático, Editorial Kapelusz. 